the short-allele carriers is probably due to increased neuronal activity that, in turn, might be caused by increased synaptic levels of serotonin. These results imply that there is a direct genetic link between serotonin receptor function and the response of a particular part of the brain to emotional information. Serotonin has been previously linked to depressive and suicidal behaviour, and the authors suggest that the amygdala might also mediate these behaviours. Such an approach could prove to be powerful in future investigations of the potential links between behavioural differences and genetic polymorphisms.

Magdalena Skipper

(4) References and links ORIGINAL RESEARCH PAPER Hariri, A. R. et al. Serotonin transporter genetic variation and the response of the human amydgala. Science $\mathbf{2 9 7}$ 400-403 (2002)

loss of one of the two visible markers in $P(w H y)$ that are on either side of hobo. A broad set of genomic deletions - which can be up to $400 \mathrm{~kb}$ but with the highest density $\sim 60 \mathrm{~kb}$ - can be generated in this way, all of which, importantly, start at one end of the hybrid element.

This deletion technique is not the first gene disruption method known in flies: RNA interference and targeted gene knockouts have been used by many labs over the past few years. What makes the $P(w H y)$ deletion system so appealing, however, is that it can be applied globally to the genome. Moreover, as the components of $P(w H y)$ have their equivalents outside Drosophila, it might not be long before this phenotypic analysis tool takes a leap into other eukaryotes.

Tanita Casci

(2) References and links ORIGINAL RESEARCH PAPER Huet, F. et al. A deletion-generator compound element allows deletion saturation analysis for genomewide phenotypic annotation. Proc. Nat/ Acad. Sci. USA 99, 9948-9953 (2002)

FURTHER READING Kornberg, T. Another arrow in the Drosophila quiver. Proc. Natl Acad. Sci. USA 99, 9607-9608 (2002)
IMMUNOGENETICS

\section{C. elegans - an innate choice?}

Much of what we know about innate immunity — an organism's immediate response to pathogen infection - comes from studies in Drosophila, which have shown that the components of innate immunity are highly conserved. Two papers now illustrate how Caenorhabditis elegans, another genetically tractable organism, might also be a useful model for studying innate immunity. Their results show that C. elegans has an inducible response to pathogen infection and that this response shares many features with innate immunity in other organisms.

Kim et al. assayed the progeny of mutagenized worms, which had been exposed to the bacterium Pseudomonas aeruginosa for enhanced susceptibility to pathogen (esp) infection. From a screen of 14,000 haploid genomes, they isolated two mutants, esp2 and $\operatorname{esp} 8$, that die much faster on exposure to $P$. aeruginosa than wild-type worms do. High-resolution SNP mapping revealed the chromosomal locations of the mutant genes, which were identified by phenotypic rescue the esp 2 mutant was rescued by the gene sek-1, and $e s p 8$ by $n s y-1$.

sek-1 encodes a MAP kinase kinase (MAPKK) homologue of mammalian MKK3/MKK6 and MKK4, and $n s y-1$ encodes an orthologue of the mammalian MAPKKK ASK1. Because these kinases activate the $\mathrm{p} 38$ kinase family and the JNK MAP kinases in mammals, the authors tested the role of $\mathrm{p} 38$ and JNK in the C. elegans defence response. The esp 2 and $e s p 8$ mutants had markedly reduced levels of p38 MAPK activity. Moreover, the knockdown of $p m k-1$, one of two C. elegans $\mathrm{p} 38$ orthologues, by RNA interference produced a strong esp phenotype. Knockdown of $p m k-2$ and a $j n k$ mutation, however, produced no enhanced susceptiblity to $P$. aeruginosa infection. Together these results show that the p38 MAPK pathway is required for innate responses to pathogen infection, which is an important discovery as this signalling pathway is also crucially required in mammals for inflammatory and innate-immune response signalling pathways.

Mallo et al. used an expression screen to look for C. elegans genes upregulated in response to infection by the bacterium Serratia marcescens. Of 7,500 cDNAs surveyed, several were induced

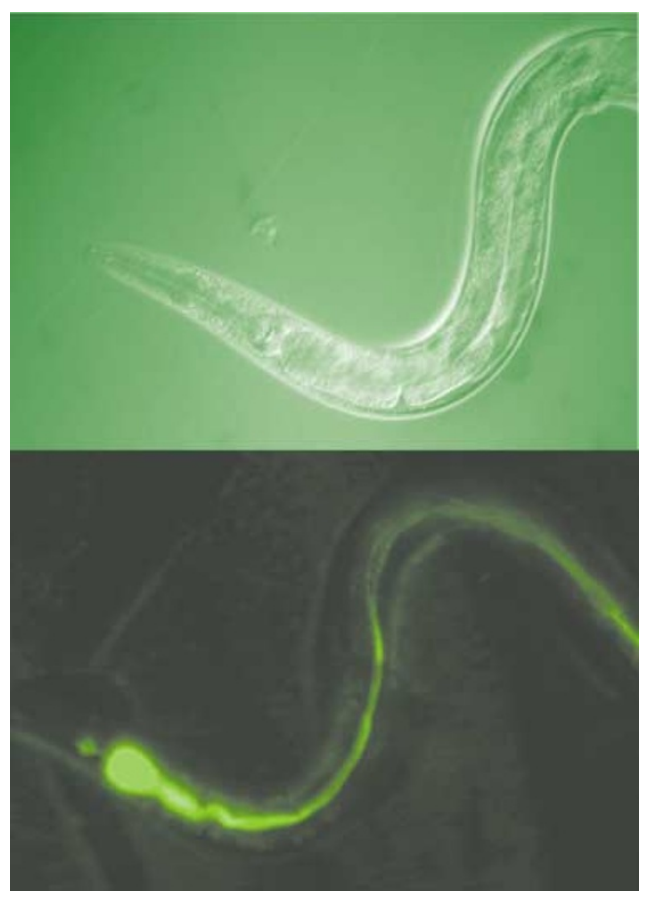

An esp2 mutant worm, after exposure to GFP-labelled $P$. aeruginosa. Nomarski (top) and fluorescence (bottom) images, courtesy of Dennis Hyong-Kun Kim, Frederick Ausubel and Rhonda Feinbaum.

over twofold, most of which encoded lectins, which function in innate immunity in other organisms. Also upregulated was lysozyme 1. As lysozymes have been implicated in innateimmune responses, Mallo et al. overexpressed $l y s-1$ in worms to see if this would enhance their resistance to $S$. marcescens. It did, although only against a less pathogenic strain of the bacterium, possibly because this strain does not produce proteases that degrade the enzyme, whereas the more pathogenic strain does. The authors also assayed $D b l-1$ mutants for their susceptibility to $S$. marcescens infection, because $D b l-1$ - a TGF- $\beta$-related gene - regulates some of the genes induced in the screen. $D b l-1$ mutants were extremely susceptible to $S$. marcescens infection and, surprisingly, also to infection by the E. coli strain OP50, which C. elegans is often cultured on.

So, 30 years after its discovery in Drosophila, these studies show that $C$. elegans also has an innate-immune response - the components of which are conserved in other organisms and the ease with which this response can be investigated genetically in worms.

60) References and links

ORIGINAL RESEARCH PAPER Kim, D. H. et al. A conserved p38 MAP kinase pathway in Caenorhabditis elegans innate immunity. Science 297, 623-626 (2002) | Mallo, G. V. et al. Inducible antibacterial defense system in C. elegans. Curr. Biol. 12, 1209-1214 (2002) FURTHER READING Kimbrell, D. \& Beutler, B. The evolution and genetics of innate immunity. Nature Rev. Genet. 2, 256-267 (2001) 